\title{
Driving Performance Profiles of Drivers with Brain Pathologies in Rural Roads
}

\author{
Dimosthenis Pavlou $^{1}$, Ion Beratis ${ }^{2}$, Panagiotis Papantoniou ${ }^{1}$ \\ Eleonora Papadimitriou ${ }^{1}$, John Golias ${ }^{1}$ and Sokratis G. Papageorgiou ${ }^{2}$ \\ ${ }^{1}$ National Technical University of Athens, Athens, Greece \\ ${ }^{2}$ National Kapodistrian University of Athens, Athens, Greece \\ dpavlou@central.ntua.gr
}

\begin{abstract}
The driving ability can be affected by various motor, visual, cognitive and perceptual deficits which are either age-related or caused by neurologic disorders. More specifically, diseases affecting a person's brain functioning may significantly impair the person's driving ability. The objective of this paper is to examine the driving performance profiles of drivers with some brain pathology in rural driving environment, in low and high traffic conditions, by means of a driving simulator experiment. Various driving performance measures are examined, e.g. mean driving speed, lateral position, steering angle, headway and reaction time at unexpected events. The driving performance of patients with brain pathologies is compared to that of healthy controls. 114 participants of above 55 years of age have completed the experimental procedure. Results suggest that there are differences between the two examined groups. Patients drive at lower speeds, present higher lateral position variability, keep larger headways and demonstrate worse reaction times than the control group.
\end{abstract}

Keywords: driving performance, brain pathologies, rural road, driving simulator experiment

\section{Introduction}

Despite the fact that road traffic casualties presented a constantly decreasing trend during the last years, the number of fatalities in road accidents in several countries in Europe and in Greece in particular is still unacceptable and illustrates the need for even greater efforts with respect to better driving performance and increased road safety [1]. A number of brain pathologies may affect driving performance in the general population and particularly in the elderly. Older drivers generally exhibit a higher risk of involvement in a road accident [2]. More specifically, diseases affecting a person's brain functioning (e.g., presence of specific brain pathology due to cerebral diseases such as Alzheimer's disease, Parkinson's disease, cerebrovascular disorders (strokes or even silent-infarcts), effect of pharmaceutical substances used for the treatment of various disturbances), may significantly impair the person's driving performance, especially when unexpected incidents occur [3-6].

Regarding Alzheimer's disease, although research findings suggest that individuals with this disease may still be fit to drive in the early stages [7], they may show visual inspection and target identification disorders during driving [8]. Moreover, the associated impairment in executive functions appears to have a significant effect on driving performance $[9,10,11]$, especially at unexpected incidents. Mild Cognitive Impairment, which is considered to be the pre-dementia stage of various dementing diseases of the brain (e.g., Alzheimer's, Cerebrovascular, Parkinson's disease), is a common disorder that may be observed in about $16 \%$ of individuals over 64 years old in the general population [12], a percentage that increases further if individuals with mild dementia are also 
included. Recent studies suggest that MCI is associated with impaired driving performance to some extent [13], as it is characterized by attentional and functional deficits, which are expected to affect the driver's ability to handle unexpected incidents. Moreover, self-reported road accident involvement was correlated with future diagnosis of dementia [14].

Neuropsychological parameters pertain to brain pathologies, as well. Because they are the neurocognitive measures of brain pathologies, they are directly linked to driving performance, in health and disease. These parameters are measured on the basis of reaction time, visual attention, speed of perception and processing, and general cognitive and executive functions. The tasks with the highest sensitivity to driving performance involve speed of visual processing, especially as measured by the Useful Field of View test, attention (e.g., selective attention, divided attention, etc.,) and executive functions $[15,16,17]$. These tasks show considerable decline with age and are associated with the probability of accident involvement $[18,19]$.

Finally, various parameters may be related to the driving performance of individuals with brain pathologies, including demographic, medical, neurological and neuropsychological parameters. However, these inter-related parameters which are rather common in the general population, especially in older adults, and may have an important effect on driving performance, especially at unexpected incidents, have not been investigated sufficiently and with a comprehensive methodology. Taking into account that the percentage of the elderly in society is increasing [20], while at the same time the level of motorization also increases [21], the need for investigation and comparative assessment of the impact of these conditions on driving performance becomes a high priority.

\section{Objectives}

The objective of this research is to examine the driving performance profiles of drivers with some brain pathology in rural driving environment, by means of a simulator experiment. The brain pathologies examined are Alzheimer's Disease (AD), Parkinson's Disease (PD), Mild Cognitive Impairment (MCI) and some others. Various driving performance measures are examined, e.g. mean speed, lateral position, steering angle, headway, reaction time at unexpected events etc. and the driving performance of patients with brain pathologies is compared to that of healthy controls of similar demographic characteristics by means of descriptive statistics. The paper starts with a presentation of a large driving simulator experiment, in which the driving performance of patients with cerebral diseases and healthy drivers was examined in different driving scenarios, followed by a thorough neurological and neuropsychological assessment of all participants. The existing sample size and characteristics are presented next, followed by a description of the results which are presented and discussed, and some concluding remarks are provided.

\section{Driving Simulator Experiment}

\subsection{Overview}

This research is based on a methodological framework for the combined assessment of traffic, behavioural, medical, neurological and neuropsychological parameters on driving performance. In this framework, the aspects of driver behaviour and safety research addressed are inherently interdisciplinary, and an experiment was designed by an interdisciplinary research team including:

- Transportation Engineers - National Technical University of Athens (NTUA)

- Neurologists - University of Athens Medical School, ATTIKON University General Hospital, Haidari, Athens 
- Neuropsychologists - Department of Psychology, University of Athens and the Aristotle University of Thessaloniki.

According to the objectives of the analysis, the experiment includes three types of assessment:

- Medical / neurological assessment: The first assessment concerns the administration of a full clinical medical, ophthalmological and neurological evaluation, in order to well document the characteristics of each of these disorders.

- Neuropsychological assessment: The second assessment concerns the administration of a series of neuropsychological tests and psychological-behavioural questionnaires to the participants. The tests carried out cover a large spectrum of Cognitive Functions: visuospatial and verbal episodic and working memory, general selective and divided attention, reaction time, processing speed, psychomotor speed etc.

- Driving at the simulator: The third assessment concerns the driving behaviour by means of programming of a set of driving tasks into a driving simulator for different driving scenarios.

The first two assessments are carried out at the ATTIKON University General Hospital, and their description is beyond the scope of this paper. The third assessment, takes place at the Laboratory of Traffic Engineering of NTUA and is presented in detail in the following section [22].

\subsection{Driving at the Simulator}

The NTUA driving simulator is a motion base quarter-cab manufactured by the FOERST Company. The simulator consists of 3 LCD wide screens 40" (full HD: 1920x1080pixels), driving position and support motion base. The dimensions at a full development are $230 \times 180 \mathrm{~cm}$, while the base width is $78 \mathrm{~cm}$ and the total field of view is 170 degrees. It's worth mentioning that the simulator is validated against a real world environment. The design of the driving scenarios of this research includes driving in rural road, in low and high traffic conditions, without external distraction. More specifically, the driving simulator experiment begins with one practice drive (usually 10-15 minutes), until the participant fully familiarizes with the simulation environment. The familiarization with the simulator includes succeeding in basic tasks regarding handling of the simulator such as braking and accelerating properly, keeping the vehicle into the lane correctly and managing to have a constant speed. Afterwards, the participant drives the rural session (approximately 20 minutes). The rural route is $2.1 \mathrm{~km} \mathrm{long,} \mathrm{single}$ carriageway and the lane width is $3 \mathrm{~m}$, with zero gradient and mild horizontal curves.

The traffic conditions examined include:

- $\mathrm{Q}_{\mathrm{L}}$-Low traffic conditions (ambient vehicles' arrivals are drawn from a Gamma distribution with mean $\mathrm{m}=12 \mathrm{sec}$, and variance $\sigma^{2}=6 \mathrm{sec}$, corresponding to an average traffic volume $\mathrm{Q}=300$ vehicles/hour)

- $\mathrm{Q}_{\mathrm{H}-\mathrm{High}}$ traffic conditions (ambient vehicles' arrivals are drawn from a Gamma distribution with mean $m=6 \mathrm{sec}$, and variance $\sigma^{2}=3 \mathrm{sec}$, corresponding to an average traffic volume of $\mathrm{Q}=600$ vehicles/hour)

Consequently, in total, each session includes two trials of the simulated route ( 2 rural road trials 2.1 each). During each trial, 2 unexpected incidents are scheduled to occur at fixed points along the drive. More specifically, incidents in rural area concern the sudden appearance of an animal (deer or donkey) on the roadway. The sequence of the trials is random in order to avoid learning effects. Finally, all patients are to carry out the 
simulator experiment while under their usual medication, so that their driving performance corresponds to their everyday condition, as treated by their neurologist.

\subsection{Participants}

For the purpose of this research 114 participants have been through the driving simulator experiment that was described analytically above. Patients and healthy controls are compared, in rural driving session, without any kind of external distraction, in low and high traffic volumes. Out of the 114 participants, 34 are controls (66.0 y.o. $\pm 8.6,17$ males), and 80 are impaired (69.0 y.o. $\pm 9.1,55$ males): $17 \mathrm{AD}$ patients (74.4 y.o. \pm 5.2$), 35$ MCI patients (70.1 y.0. \pm 5.0$), 16$ PD patients (66.1 y.o. \pm 7.6$)$ and 12 patients of other brain pathologies (66.2 y.o. \pm 6.4 ). It is worth mentioning that all 114 participants are above 55 years of age. This was carefully selected in order not to have age as a parameter that may affects the results, but only their cerebral condition.

\subsection{Key Driving Performance Measures}

The key driving performance measures examined in this research correspond to longitudinal and lateral driving control measures and are presented below:

- mean speed (mean speed of the driver along the route, excluding the small sections in which incidents occurred, and excluding junction areas)

- time headway (time space between the simulator vehicle and the vehicle ahead, excluding the small sections in which incidents occurred, and excluding junction areas)

- reaction time (time between the first appearance of the event - "hazard" on the road and the moment the driver starts to brake)

- steering angle (average steering angle of the wheel, excluding the small sections in which incidents occurred, and excluding junction areas)

- lateral position (distance between the vehicle and the middle axis of the road, excluding the small sections in which incidents occurred, and excluding junction areas)

All these key measures were analyzed by descriptive statistics and the results are presented below. It is worth mentioning that mean values are compared and average values plus and minus standard deviation are shown on diagrams.

\section{Results}

\subsection{Mean Speed}

In Figure 1, the mean speed of drivers along the trial (in rural road area, in high $\left(\mathrm{Q}_{\mathrm{H}}\right)$ and low $\left(\mathrm{Q}_{\mathrm{L}}\right)$ traffic volume) is presented per driving group. It is observed that control drivers drove the trial road section at approximately $16 \%$ higher speed than impaired drivers in both low and high traffic volumes. Mean speed is lower in high traffic volume conditions, as expected. Finally, the variability of speed is lower in high traffic volume as expected due to the dense traffic conditions. 


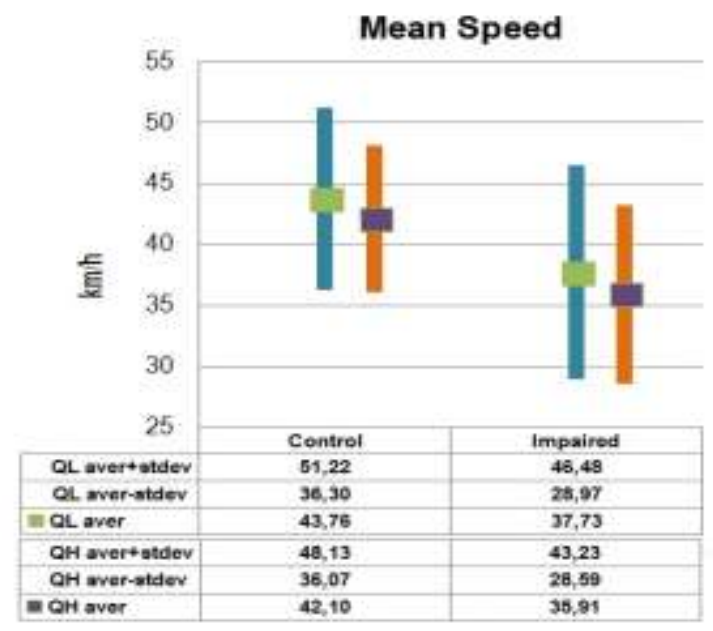

Figure 1. Mean Speed $(\mathrm{km} / \mathrm{h})$

(blue column refers to low traffic, orange column refers to high traffic)

\subsection{Time Headway}

In Figure 2, the time headway of drivers is presented for the examined conditions. It is observed that impaired drivers keep larger headways from the vehicle ahead compared to the control group (52\% in low traffic volume and $66 \%$ in high traffic volume). This is obviously happening because of their lower speed and their conservative driving. Impaired drivers seem to having trouble dealing with the high traffic volume and their time headways are significally reduced. Of course in high traffic volume lower time headways are obvious results. It's worth noticing the large variability of mean space headways for impaired drivers in both traffic volumes compared with the variability of the control group headways.

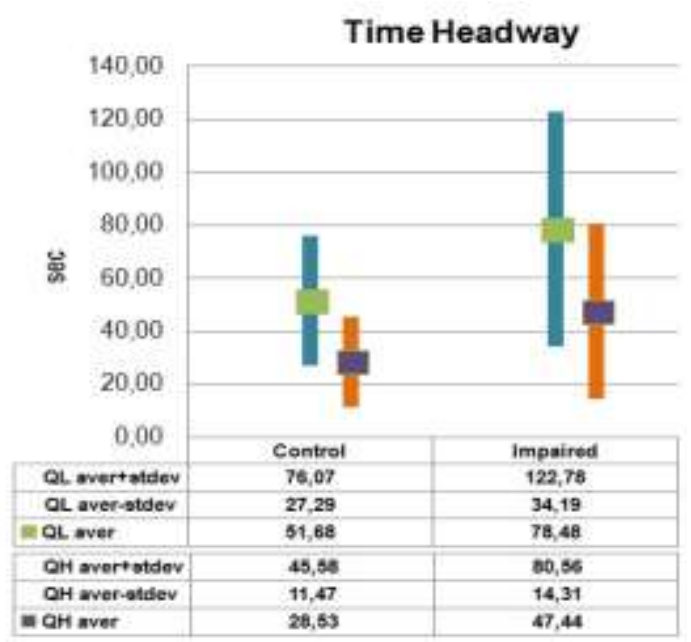

Figure 2. Time Headway (sec)

(blue column refers to low traffic, orange column refers to high traffic)

\subsection{Lateral Position}

In Fig. 3, the lateral position of drivers is presented. Lateral position profiles of both examined groups don't seem to have any significant differences. It is observed that in high traffic volume both control and impaired drivers drive approximately 10\% more closely to the right border of the road. 


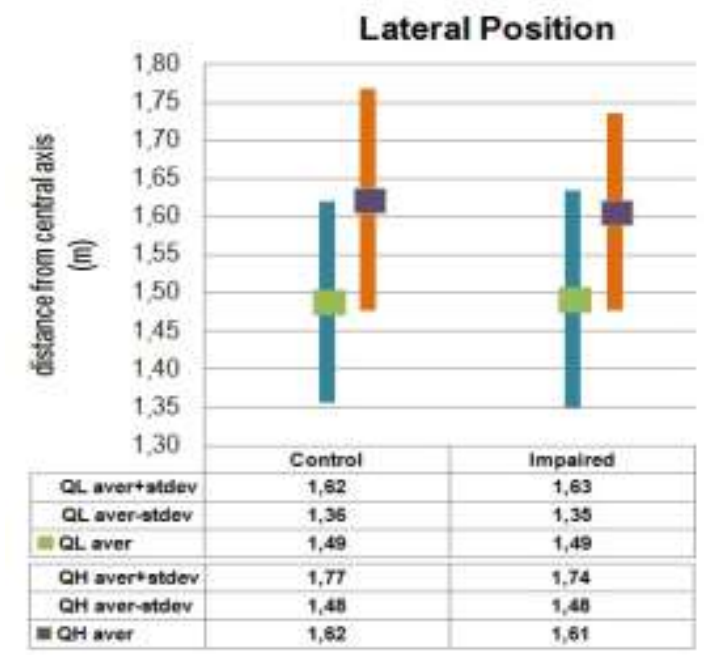

Figure 3. Lateral position ( $m$ )

(blue column refers to low traffic, orange column refers to high traffic)

\subsection{Steering Angle}

In Figure 4, steering wheel is examined. It is worth mentioning that lower steering angle means wheel to the right. It is observed that no significant differences are presented between control and impaired group. In high traffic volumes all participants tend to drive at the right border of the road (this was observed in lateral position results too). Finally, the higher the traffic is, the more variability in wheeling angle the control drivers have, whereas the lower traffic volume is, the more variability in wheeling angle the impaired drivers have.

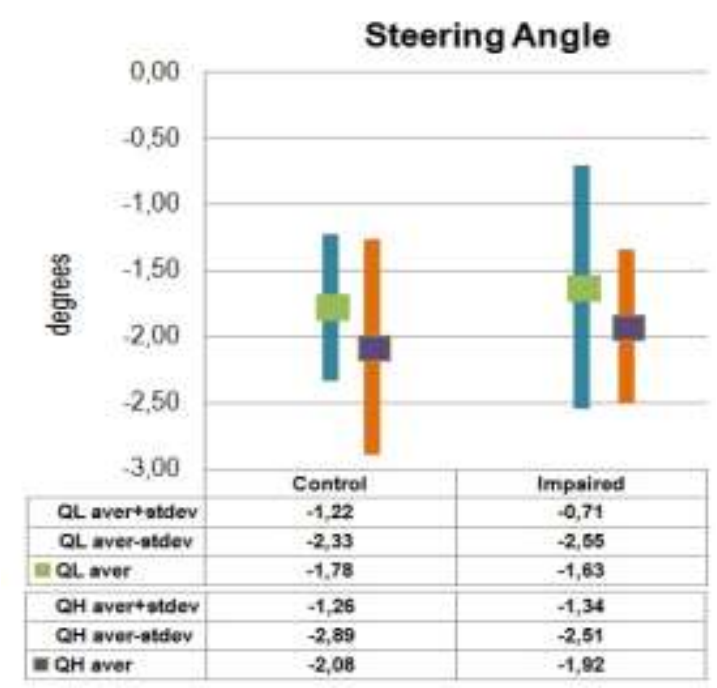

Figure 4. Steering Angle (degrees)

(blue column refers to low traffic, orange column refers to high traffic)

\subsection{Reaction Time}

In Figure 5, the reaction time of drivers is presented per driving condition. Impaired drivers have worse reaction times than the control ones (30\% worse overall). These worse reaction times of impaired drivers are confirmed by their neurological and neuropsychological assessment. At the present time the medical and neuropsychological database is under preparation in order to be finalized and used in future statistical 
analyses, and thus it is not available. Finally, traffic volume appears to affect the reaction time of all drivers.

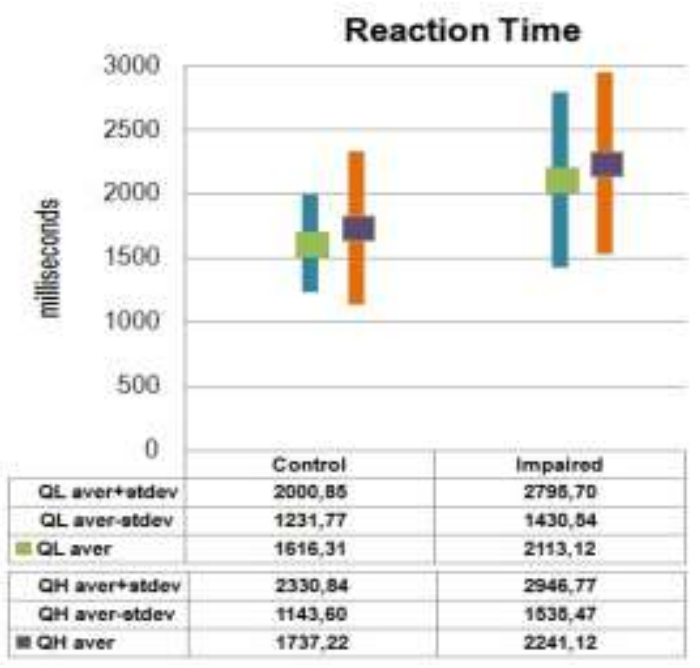

Figure 5. Reaction Time (millisec)

(blue column refers to low traffic, orange column refers to high traffic)

\subsection{Speed Profiles}

In Figure 6, the average speed profiles of drivers along the rural road trial (low and high traffic volumes, no external distraction) are presented per driver condition. It is observed that all drivers present very similar speed profiles in terms of general shape, which is intuitive given that the shape of the speed profile is largely dependent on the road geometry, the appearance of incidents (i.e. speed drop is visible at around $\mathrm{Km} 1.05$ and $\mathrm{Km} 1.35$ of the profile) and the traffic conditions, which were the same for all drivers.

Healthy drivers drove the trial road section at higher speeds than impaired drivers, which is also arising from chapter 4.1. It is also observed that the difference between the two traffic volumes is obvious in both examined groups.

Moreover, it is observed that after the first unexpected incident impaired drivers are more careful and don't seem to reach the speed they had before the incident, which is a compensatory strategy, in order to self-regulate their driving behaviour.

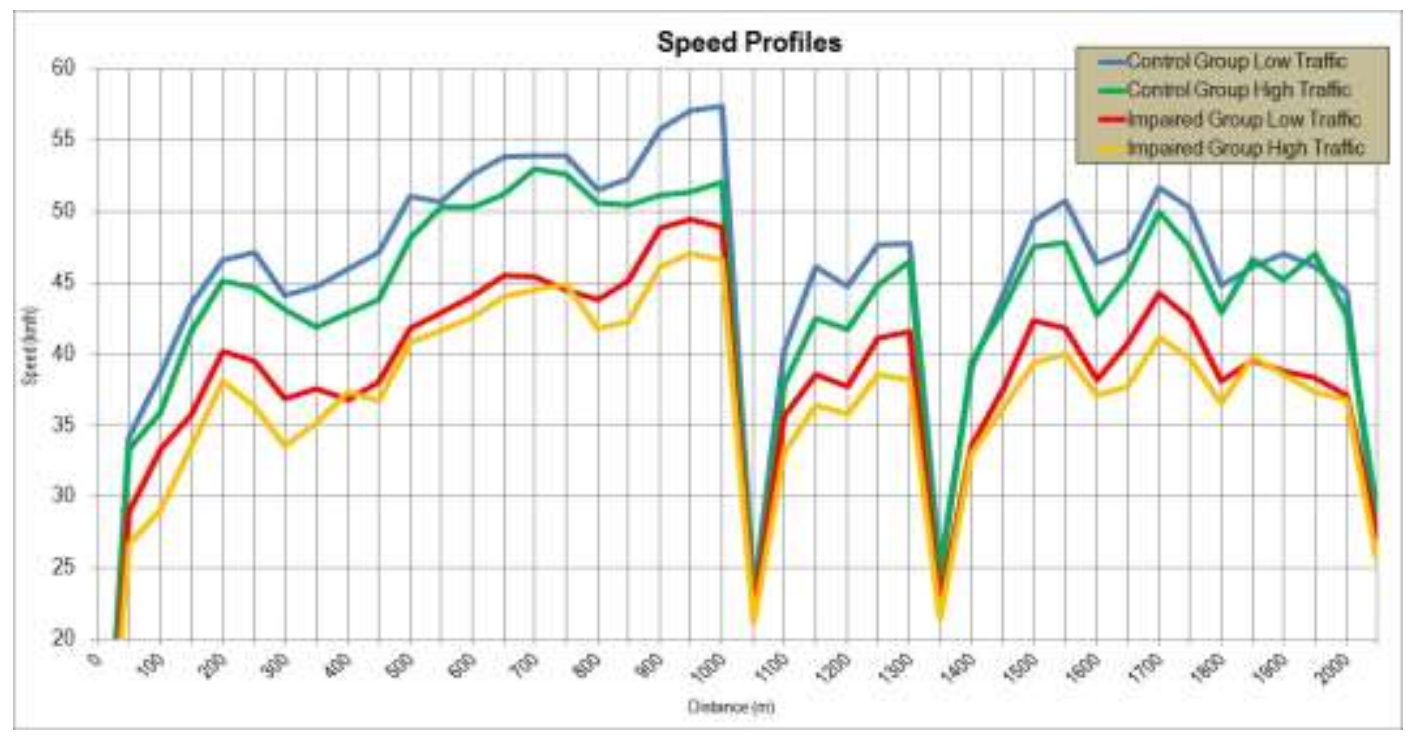

Figure 6. Average Speed Profiles of Examined Groups 
In Figure 7 and Figure 8, speed profiles of all participants are presented. We can detect overall higher speeds for healthy drivers compared to the impaired group's speeds in both traffic volumes. This finding though was detectable from the chapter 4.1.

It is observed that impaired group (red lines in low traffic figure and yellow lines in high traffic figure) has variability between 20 and $60 \mathrm{~km} / \mathrm{h}$, whereas control group (blue lines in low traffic figure and green lines in high traffic figure) has a smaller variability between 40 and $70 \mathrm{~km} / \mathrm{h}$.

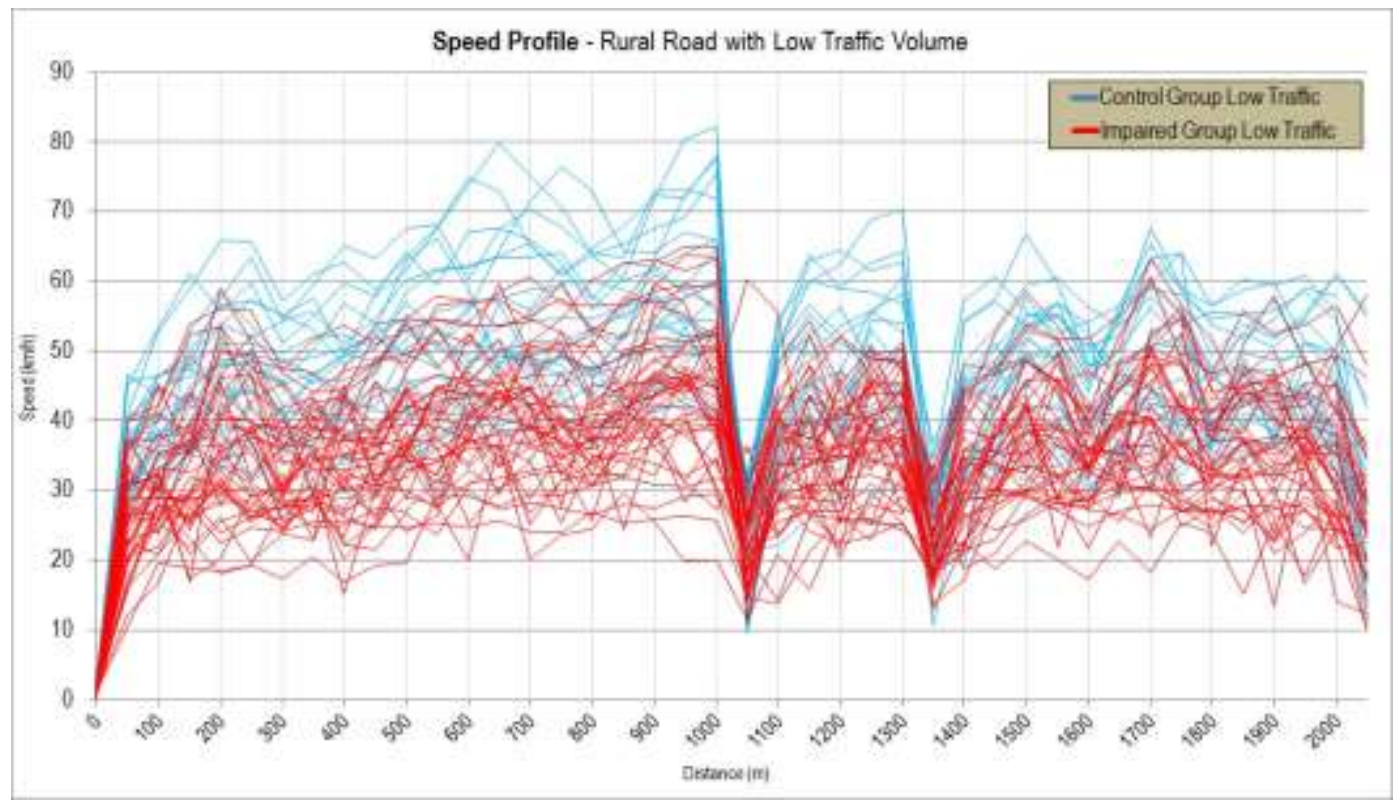

Figure 7. Speed Profiles of all Drivers in Low Traffic Volume

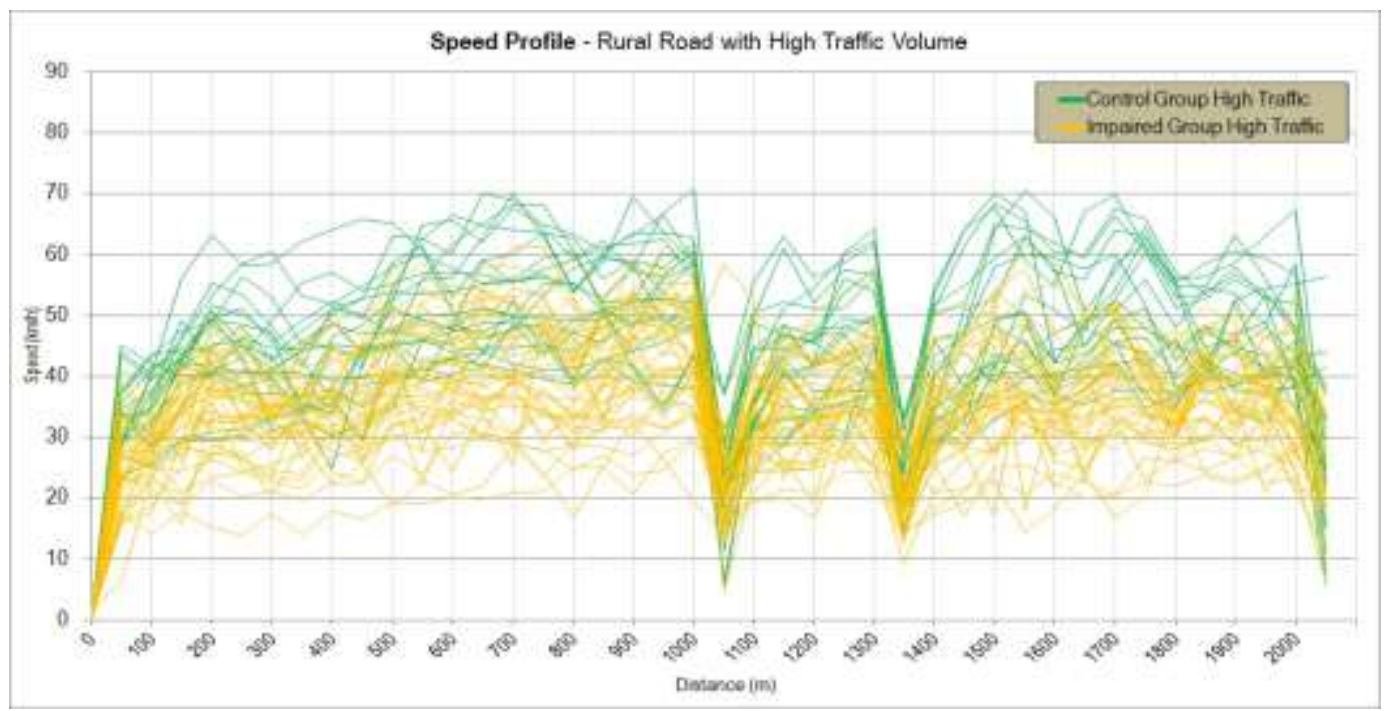

Figure 8. Speed Profiles of all Drivers in High Traffic Volume

\subsection{Lateral Position Profiles}

Figure 9 concerns the mean lateral position profiles per driver group along the rural road section trial (low and high traffic volumes, no external distraction). Lateral position is defined as the vehicle's distance from the central axis of the road. In this case, differences between drivers are less pronounced, yet detectable. Lateral position profiles between healthy drivers and impaired drivers exhibit more similarities in terms of overall 
shape of the profile and magnitude of the examined measure. It seems that high traffic conditions lead drivers to drive to the right border of the road. These smaller differences in lateral position between healthy and impaired drivers may be attributed to the fact that, by definition, lateral position is a less sensitive measure of driving performance (due to the restrictions of the road section geometry).

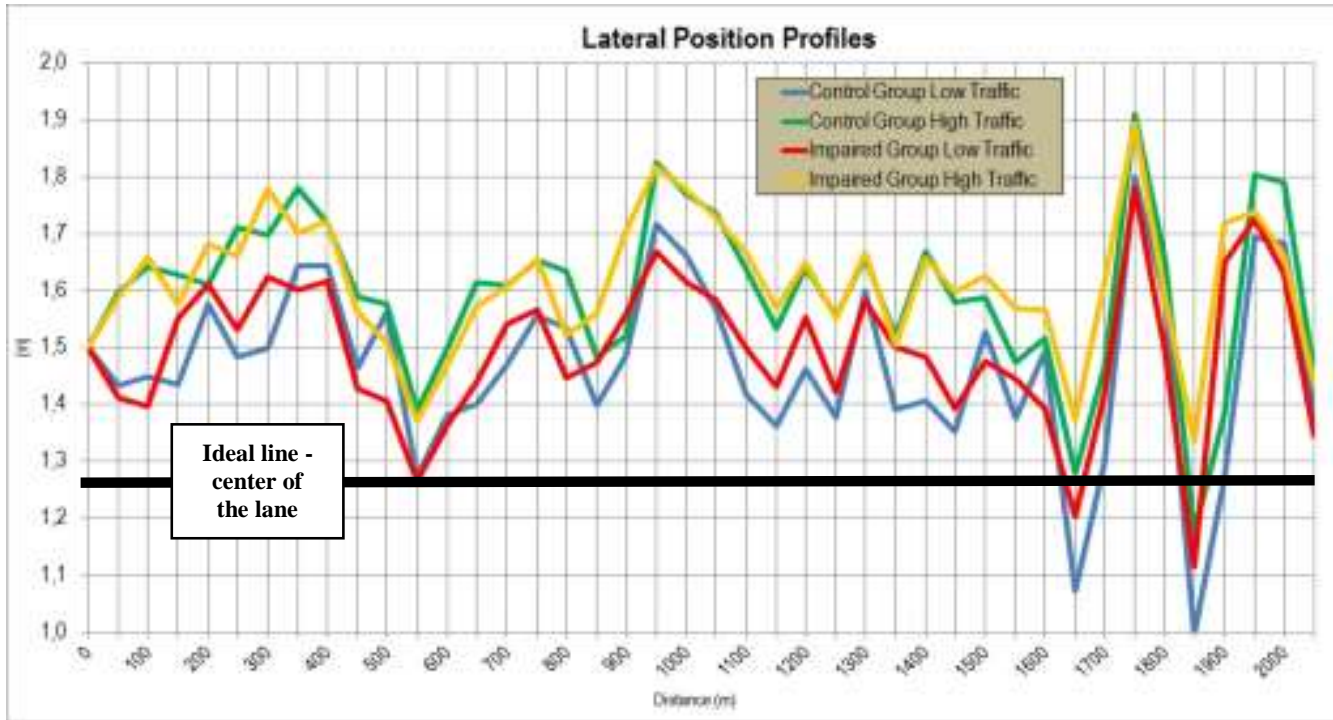

Figure 9. Average Lateral Position Profiles of Examined Groups

Moreover, after $1650 \mathrm{~m}$ we detect a big variability of lateral position. This is happening due to the fact that in this particular segment of the route there is the only "demanding" turn of the trial (quick left turn followed by quick right turn). In Figure 10 and Figure 11, lateral position profiles of all participants are presented. In overall, it is observed that in both traffic volumes has higher variability in lateral position (more "red" and "yellow" peaks in he figures than "blue" and "green" ones). This happens because of the difficulty the impaired group has to keep a smooth driving line along the route.

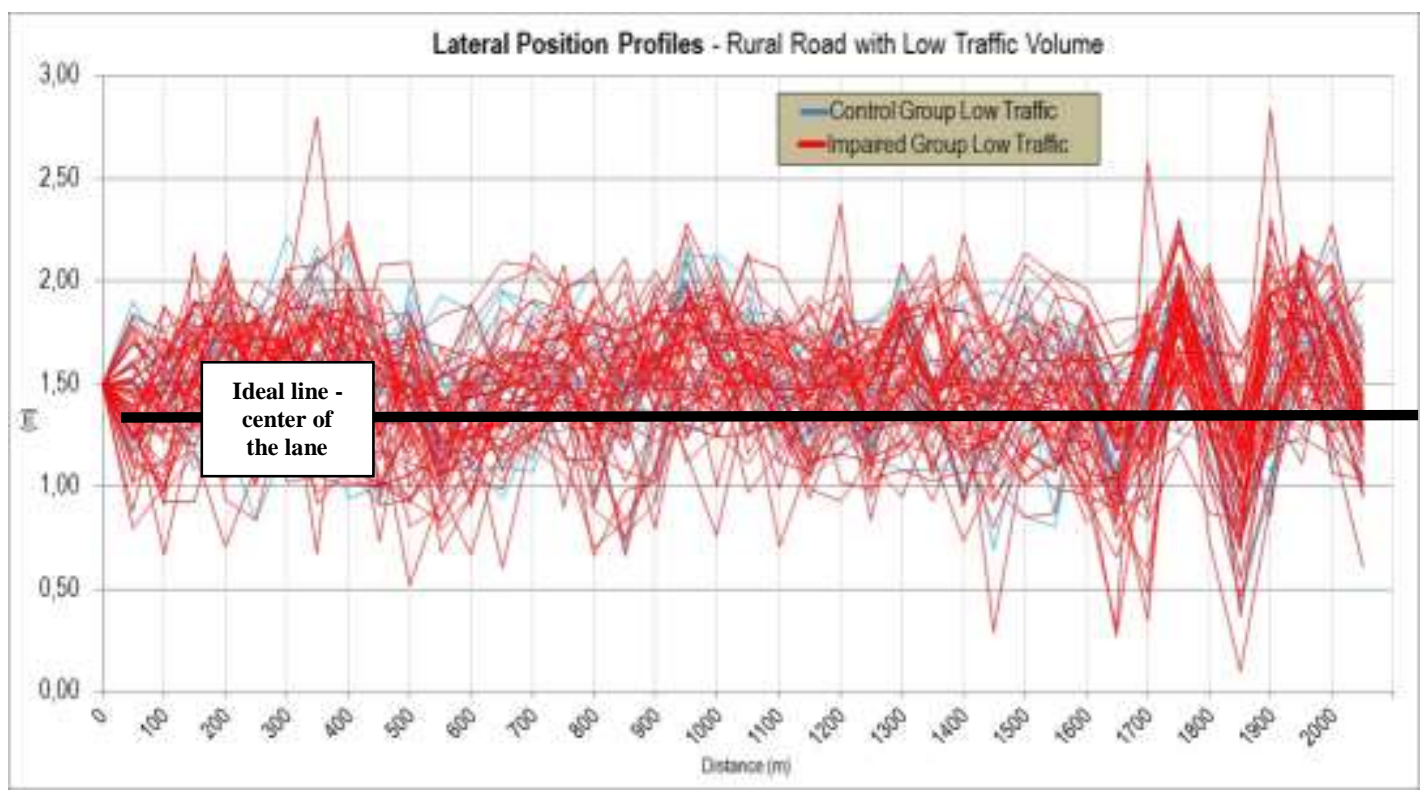

Figure 10. Lateral Position Profiles of all Drivers in Low Traffic Volume 


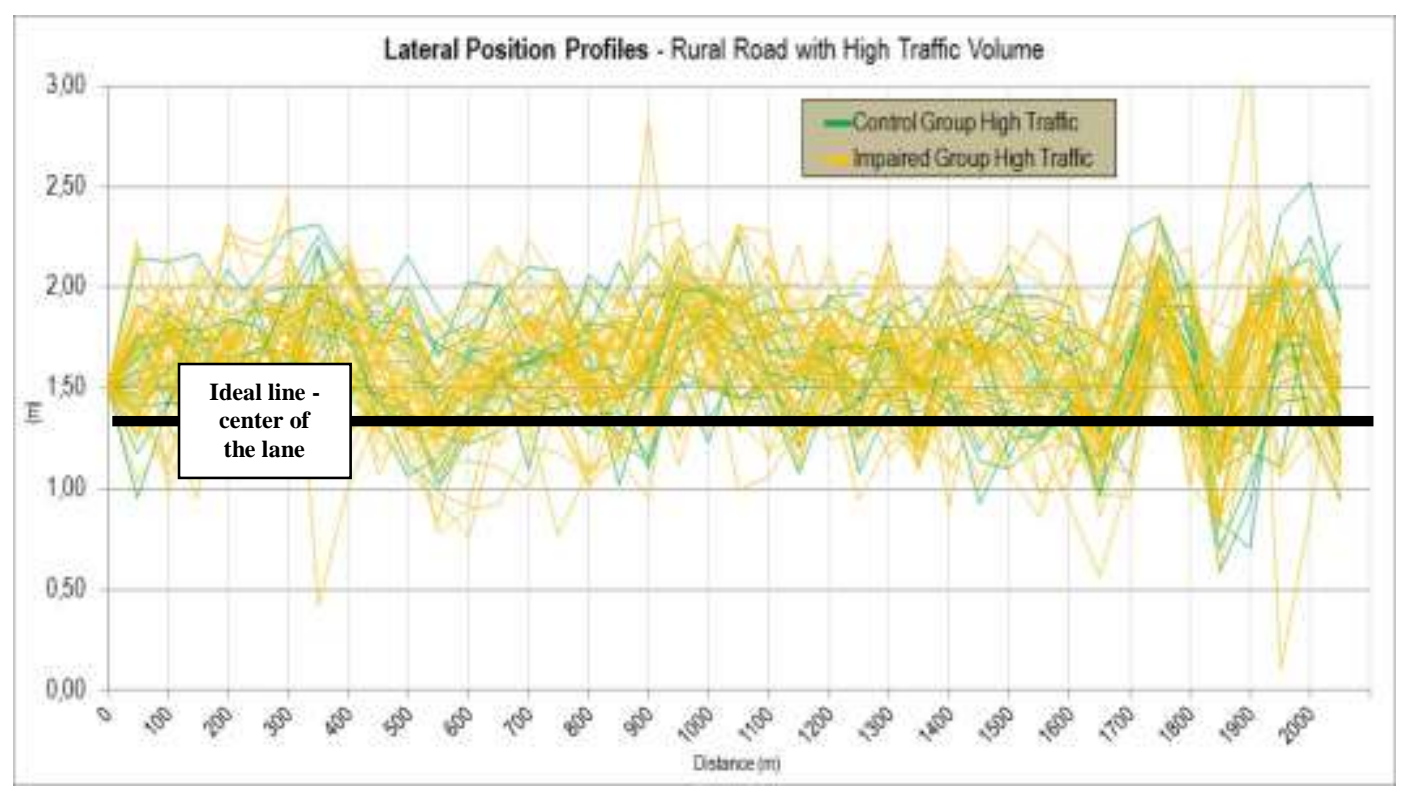

Figure 11. Lateral Position Profiles of all Drivers in High Traffic Volume

\section{Conclusions and Discussion}

The objective of this paper was to present and analyze the driving performance profiles of drivers with some brain pathology in rural driving environment, by means of a simulator experiment. The brain pathologies examined were Alzheimer's disease (AD), Parkinson's disease (PD), Mild Cognitive Impairment (MCI) and some other brain pathologies. Various driving performance measures were examined, e.g., mean speed, lateral position, steering angle, headway, reaction time at unexpected events etc. and the driving performance of drivers impaired by brain pathologies was compared to that of healthy controls by means of descriptive statistics. More specifically, 114 participants (34 controls, 80 impaired, all above 55 years of age) had been through the driving simulator experiment. Impaired and Control group were compared, in rural driving session, without any kind of external distraction, in low and high traffic volume.

Results suggested difficulties in driving performance of the impaired group in almost every driving performance measure examined. More specifically, regarding the driving speed, and its variability, it was observed that control drivers drove at approximately $16 \%$ higher speed than impaired drivers in both low and high traffic volumes and have lower variability in their speed. The speed profiles of the participants confirm these results. Then, examining the headway, it was observed that due to the lower speeds, impaired drivers kept much larger headways (more than 50\% larger) which was expected. It is worth mentioning, though, the large variability in impaired drivers' headways along the driving route. This means that they could not adjust their speed and had significant difficulties in keeping constant and safe headways.

The lateral position results appeared to be the same between the two groups, but the lateral position profiles that were extracted, presented a large variability in impaired drivers' lateral position along the route, which means difficulties in positioning the vehicle properly in the lane. It is worth noticing that in high driving conditions all examined groups drove more closely to the right border of the road.

Moreover, regarding the steering wheel, it was observed that the higher the traffic was, the more variability in steering angle the control drivers had, whereas the lower traffic volume was, the more variability in wheeling angle the impaired drivers had. A possible explanation is that in high traffic volumes healthy drivers may try to overtake the vehicle ahead and thus they have variability in wheeling angle, whereas impaired drivers (who 
drive at lower speeds) may use the vehicle ahead as a "guide" and just drive behind them conservatively, following a self-regulation strategy regarding their driving performance. In every case, this finding is very interesting and will be examined in the future in a larger sample.

Finally, drivers suffering from brain pathology had significantly larger reaction times in both traffic environments compared to the control group (almost 30\% worse reaction times). This finding may lead to higher accident probability.

All above results are quite promising and confirm the initial hypotheses of the research that brain pathologies may deteriorate driving performance in several ways regarding road safety. Further statistical analyses, though, with a larger sample scheme will strengthen our study. Finally, the results are to be considered within the limiting context of driving simulator studies - driving performance is known to be more accurately and reliably estimated by means of on-road studies. However, the relative effects of impaired vs. healthy drivers are known to be quite identifiable in simulator studies.

\section{Acknowledgments}

This paper is based on a research project implemented within the framework of the Operational Program "Education and Lifelong Learning" of the National Strategic Reference Framework (NSRF) - Research Funding Program: THALES. Investing in knowledge society through the European Social Fund, and is co-financed by the European Union (European Social Fund - ESF) and Greek national funds.

\section{References}

[1] OECD, Towards Zero: Ambitious Road Safety Targets and the Safe System Approach. OECD, Paris, (2008) October.

[2] M. R. J. Baldock, J. Mathias, J. McLean and A. Berndt, "Visual attention as a predictor of on-road driving performance of older adults", Australian Journal of Psychology, vol. 59, (2007), pp. 159-168.

[3] J. M. Wood and C. Worringham, "Quantitative assessment of driving performance in Parkinson's disease", Journal of Neurol Neurosurg Psychiatry, vol. 76, no. 2, (2005), pp. 176-80.

[4] R. Cordell and H. C. Lee, "Driving assessment in Parkinson's disease - a novel predictor of performance?", Mov Disord., vol. 23, no. 9, (2008), pp. 1217-22.

[5] E. Cubo and P. Martinez Martin, "What contributes to driving ability in Parkinson's disease", Disabil Rehabil, (2009).

[6] C. Frittelli, D. Borghetti, G. Iudice, E. Bonanni, M. Maestri, G. Tognoni, L. Pasquali and A. Iudice, "Effects of Alzheimer's disease and mild cognitive impairment on driving ability: a controlled clinical study by simulated driving test", International Journal of Geriatric Psychiatry, vol. 24, (2009), pp. 232238.

[7] B. R. Ott, W. C. Heindel, W. M. Whelihan, M. D. Caron, A. L. Piatt and M. A. DiCarlo, "Maze test performance and reported driving ability in early dementia", Journal of Geriatric Psychiatry and Neurology, vol. 16, (2003), pp. 151-155.

[8] E. Uc, and M. Rizzo, "Driving in Alzheimer's Disease, Parkinson's Disease and Stroke", In: Fisher, D., Rizzo, M., Caird, J., Lee J., Handbook of Driving Simulation for Engineering, Medicine and Psychology, CRC Press, (2011).

[9] D. Pavlou, E. Papadimitriou, G. Yannis, P. Papantoniou and S. G. Papageorgiou, "First findings from a simulator study on driving behaviour of drivers with cerebral diseases", Proceedings of the Transport Research Arena Conference, Paris, (2014).

[10] E. Papadimitriou, D. Pavlou, P. Papantoniou, G. Yannis, J. Golias and S. G. Papageorgiou, "Results from a driving simulator study on performance of drivers with cerebral diseases in rural roads", Proceedings of the 93rd Annual meeting of the Transportation Research Board, Washington, (2014).

[11] H. Tomioka and B. Yamagata, "Detection of hypofrontality in drivers with Alzheimer's disease by nearinfrared spectroscopy", Neurosci Lett., vol. 451, no. 3, (2009), pp. 252-6.

[12] G. Ravaglia, P. Forti, F. Montesi, A. Lucicesare, N. Pisacane and E. Rietti, "Mild Cognitive Impairment: Epidemiological and dementia risk in an elderly Italian population", Journal of the American Geriatrics Society, vol. 56, (2008), pp. 51-58.

[13] C. Frittelli, D. Borghetti, G. Iudice, E. Bonanni, M. Maestri, G. Tognoni, L. Pasquali and A. Iudice, "Effects of Alzheimer's disease and mild cognitive impairment on driving ability: a controlled clinical study by simulated driving test", International Journal of Geriatric Psychiatry, vol. 24, (2009), pp. 232238. 
[14] S. Lafont, B. Laumon, C. Helmer, J. F. Dartigues and C. Fabrigoule, "Driving cessation and self-reported car crashes in older drivers: The impact of cognitive impairment and dementia in a population-based study", Journal of Geriatric Psychiatry and Neurology, vol. 21, (2008), pp. 171-182.

[15] L. A. Bieliauskas, "Neuropsychological assessment of geriatric driving competence", Brain Injury, vol. 19 , pp. 221-226.

[16] J. L. Mathias and L. K. Lucas, "Cognitive predictors of unsafe driving in older drivers: A meta-analysis", International Psychogeriatrics, vol. 21, (2009), pp. 637-653.

[17] B. Weaver, M. Bédard, J. McAuliffe and M. Parkkari, "Using the Attention Network Test to predict driving test scores", Accident Analysis and Prevention, vol. 41, (2009), pp. 76-83.

[18] O. Clay, V. G. Wadley, J. D. Edwards, D. L. Roth, D. L. Roenker and K. Ball, "Cumulative metaanalysis of the relationship between useful field of view and driving performance in older adults: Current and future implications", Optometry and Vision Science, vol. 82, (2005), pp. 724-731.

[19] M. Lunsman, J. D. Edwards, R. Andel, B. J. Small, K. K. Ball and D. L. Roenker, "What predicts changes in Useful Field of View test performance?", Psychology and Aging, vol. 23, (2008), pp. 917-927.

[20] M. R. J. Baldock, J. Mathias, J. McLean and A. Berndt, "Visual attention as a predictor of on-road driving performance of older adults", Australian Journal of Psychology, vol. 59, pp. 159-168.

[21] G. Yannis, C. Antoniou, E. Papadimitriou and D. Katsohis, "When may road fatalities start to decrease?", Journal of Safety Research, vol. 42, no. 1, (2011), pp. 17-25.

[22] G. Yannis, J. Golias, C. Antoniou, E. Papadimitriou, S. Vardaki, P. Papantoniou, D. Pavlou, S. Papageorgiou, N. Andronas, I. Papatriantafyllou, A. Liozidou, I. Beratis, D. Kontaxopoulou, S. Fragkiadaki and A. Economou, "Design of a large driving simulator experiment on performance of drivers with cerebral diseases", Proceedings of the 4th International Conference on Road Safety and Simulation, (2013).

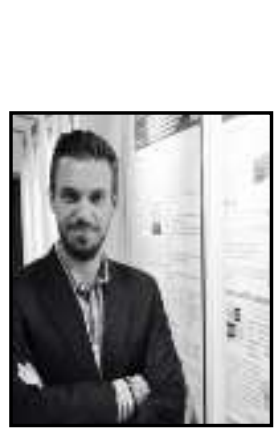

\section{Author}

Dimosthenis Pavlou, is a Civil Engineer, PhD Candidate and Researcher at the Department of Transportation Planning and Engineering at the School of Civil Engineering of the National Technical University of Athens.

In 2008, he graduated from the School of Civil Engineering at the NTUA after having followed the option of Structural Engineering. He has a Master of Science in the Interdisciplinary - Interdepartmental Program "Analysis and Design of Earthquake Resistant Structures".

Currently he is a PhD Candidate and Researcher in the Department of Transportation Planning and Engineering in NTUA in the field of traffic safety. He has participated in 3 research projects, he has published 1 Diploma Thesis and 1 Master Thesis, 17 conference papers, and he has attended several scientific conferences.

Dimosthenis Pavlou (Corresponding author)

MSc, PhD Candidate, Research Assistant

National Technical University of Athens

Department of Transportation Planning and Engineering

5 Heroon Polytechniou st., GR-15773 Athens

Tel: +302107721380, Fax: +302107721454

E-mail: dpavlou@central.ntua.gr 